\title{
Variational technique for gauge boson masses
}

\author{
B. $\operatorname{Koch}^{1,2, *}$ and C. Laporte ${ }^{2,3, \dagger}$ \\ ${ }^{1}$ Institute for Theoretical Physics, TU Wien, Wiedner Hauptstrasse 8, A-1040 Vienna, Austria \\ ${ }^{2}$ Instituto de Física, Pontificia Universidad Católica de Chile, Avenida Vickuña Mackenna 4860, Santiago, Chile \\ ${ }^{3}$ Institute for Mathematics, Astrophysics and Particle Physics (IMAPP), Radboud University Nijmegen, \\ Heyendaalseweg 135, 6525 AJ Nijmegen, The Netherlands
}

(Received 24 March 2020; accepted 23 January 2021; published 15 February 2021)

\begin{abstract}
In this work, a novel mechanism for spontaneous symmetry breaking is presented. This mechanism incorporates gravity in the generation of masses for gauge fields, showing that the Higgs mechanism can take place even without a quartic self-interaction at the fundamental level. Using the scale-dependent effective action $\Gamma_{k}$ minimally coupled to a gravitational sector, a variational parameter setting is applied. This provides a mass and vacuum expectation value as a function of the constants arising in the low-scale expansion of Newton and cosmological couplings. A comparison with experimental data, such as the Higgs mass, allows putting restrictions on these constants. This generic approach can be compared with explicit candidates for an effective field theory of gravity. As an example, we use the asymptotic safety scenario, where we find restrictions on the matter content of the theory.
\end{abstract}

DOI: 10.1103/PhysRevD.103.045011

\section{INTRODUCTION}

The Standard Model describes the elementary particles and their interactions in a successful way. However, it does not contain a description of gravitational interactions, and there are some problems with the natural implementation of spontaneous symmetry breaking (SSB) [1-6].

\section{A. The idea of gravity-assisted emergent Higgs mechanism in flat spacetime}

There is no doubt that SSB plays an essential role in our modern theoretical physics conception. A wide number of phenomena, such as the spatial order of ferromagnets $[7,8]$, convecting fluids $[9,10]$, and superconductivity $[11,12]$, are understood by means of SSB. It is also crucial in the Standard Model (SM) of elementary particles. Here, the mechanism is responsible for generating the masses of elementary particles [13-15].

The most straightforward realization of a gauge field theory incorporating SSB is coupling the gauge and fermion fields with a scalar field $\Phi$ with negative squared mass and quartic self-interaction. Thus, the potential acquires a nontrivial vacuum expectation value (VEV),

\footnotetext{
*bkoch@puc.uc.cl

calaporte@uc.cl
}

Published by the American Physical Society under the terms of the Creative Commons Attribution 4.0 International license. Further distribution of this work must maintain attribution to the author(s) and the published article's title, journal citation, and DOI. Funded by SCOAP. breaking the underlying symmetry. The scalar field's components in the direction of the broken symmetry become the longitudinal components of the gauge bosons. At the same time, the remaining elements convert to what we know as the Higgs particle. Without it, the theory would violate unitarity.

In general, gravity is of negligible importance in the elementary particle's arena, in particular, in the way SSB generates masses for gauge bosons. The general assumption of ignoring gravitation in particle interaction is well justified in the theoretical point of view, because gravitational contributions are highly suppressed in the renormalization group (RG) flow of the couplings that govern the low-energy regime. Still, the fact that only the Higgs boson carries the mass scale into an otherwise scale-free SM, and thus tells the SM particles how they should gravitate, means that the two branches are not entirely disconnected.

In this work, a novel way of spontaneously breaking the Higgs field's symmetry when the scalar sector does not possess self-interaction is presented. A dynamically induced Higgs-like SSB scalar potential was obtained by considering a model of gravity interacting minimally with a scalar quantum electrodynamics (QED) without a quartic term in the scalar field. As we see below, a vacuum energy density introduces an effective quartic interaction when all the unphysical parameters of the theory have been removed. Correspondingly, the new mechanism for SSB introduces gravity in the production of masses to elementary particles such that they do not depend on unphysical parameters, retain diffeomorphism invariance, and are insensitive to UV contributions from the gravitational sector of this theory. 
While the method for inducing SSB in this work is (as far as we know) new, there have been some previous studies on SSB in models that do not show SSB explicitly in the matter sector of the theory [16-22]. These ideas are based on the use of a gravity-assisted emergent Higgs mechanism. As a consequence, the process of generating a Higgs-like potential generally involves a scalar field coupled to the curvature invariant of the Riemannian manifold $[16,17,20]$ or a connection field, without a Higgs boson [18]. Thus, in these theories SSB can take place only in curved spacetime, where, for example, the curvature scalar $R$ is of the order of a cosmological constant $\Lambda_{0}$. For an implementation in mark of the SM, this is not practical, since today's value of the cosmological constant $\Lambda_{0}$ is known to be extremely small. Even though the presented mechanism starts from a gravitational sector with a cosmological term, the infrared value of the cosmological constant $\Lambda_{0}$ does not play a role in the SSB mechanism. All the results obtained in this work are valid in the limit where the Ricci curvature goes to zero. Thus, the presented model SSB is well defined even in flat spacetime.

\section{B. The idea of SSB without quartic interaction}

The goal of this paper is to point out a novel way of inducing spontaneous symmetry breaking. It allows generating masses for gauge bosons without self-interaction terms of scalar fields in the bare action. This mechanism avoids the presence of unphysical parameters at the physical observables while it provides a novel way of producing SSB, taking into account the gravitational sector.

The starting point will be a bare action without interactions like the quartic Higgs coupling. Quantum corrections to this classical bare action yield a scale dependence at the level of the gauge couplings contained in an effective action $\Gamma_{k}$. The arbitrary renormalization scale cannot be part of physical observables. It will be set following the variational parameter setting (VPS) [23] prescription, which can be understood as the principle of minimal sensitivity [24], applied to quantum field theory background calculations. The VPS prescription allows minimizing the scale-dependent effective action with respect to variations of its field and parameter content. Variations with respect to the field content give the wellknown gap equations [25], which are complemented by one, variationally derived, scale-setting equation.

It is possible to choose a bare action $S(\Phi)$ where a massive scalar interacts only minimally with the metric field, without self-interactions. This means, for constant values of the renormalization scale $k$, there will be no terms, which are usually necessary to generate SSB. However, in every quantum field theory calculation, the scale $k$ has to be set in order to arrive at a testable prediction. This necessity is a consequence of the incomplete nature of any perturbative or effective quantum field theory approach. It depends on the observable one is interested in and whether one chooses $k$ as a function of kinematic variables, renormalized parameters, or something else. Thus, assuming the scale to be an independent constant was actually inconsistent. For example, when one is interested in background configurations, one way to proceed is the scale setting of the "improving solutions" procedure, leading to Uehling-type potentials [26-37]. However, the scale setting in improving solutions procedures leads to an anomalous violation of the underlying gauge symmetries. Fortunately, such a breaking of gauge symmetries is not necessary. As shown in Ref. [23], the aforementioned VPS prescription allows one to derive an optimal scale setting $k \rightarrow k_{\mathrm{opt}}$, which preserves the underlying gauge symmetries of the effective action $\Gamma_{k}$. After the replacement $k \rightarrow k_{\mathrm{opt}}$, the effective action $\Gamma_{k}$ becomes an optimal effective action $\Gamma_{\mathrm{opt}}$.

The main message of this paper is that, for SSB to occur, it is sufficient that it occurs at the level of this optimal effective action $\Gamma_{\text {opt }}$, as opposed to the nonoptimal effective action $\Gamma_{k}$ or the bare action $S$. First, the quadratic divergences arising from quantum corrections of the bare action $S$ in the standard SSB are absent when SSB occurs only at the level of $\Gamma_{\mathrm{opt}}$, since the optimal effective action has all quantum corrections already incorporated $[38,39]$. Thus, no additional potentially dangerous quantum corrections are necessary. Second, gravitationally induced SSB can be implemented, even for flat background spacetime.

This idea is conceptually appealing. In Sec. II, it is implemented for scalar QED. It turns out that the resulting optimal effective action $\Gamma_{\text {opt }}$ allows for SSB only if the gauge fields form a condensate. Even though this might be an interesting possibility, it deviates from our original intention. One realizes that, for the program to work, one needs a scaledependent vacuum contribution to the effective action. This is the reason why we proceed with an effective description of quantum gravity, where this vacuum contribution is given in terms of a cosmological constant. As an example, we study the asymptotic safety (AS) approach. The AS [40] conjecture provides a consistent description of gravity as a nonperturbatively renormalizable quantum field theory [41-43] and a scenario for testing the results of this work. Moreover, the scale dependence of the gravitational and cosmological constant has been extensively studied in Refs. [44-50] as well as properties and consequences of the scale-dependent Einstein-Hilbert action [51-56] and Gaussian massless matter fields minimally coupled to an external metric [57-64].

The paper is organized as follows: Below, a discussion of similarities and differences with the Coleman-Weinberg (CW) mechanism [65]. In Sec. II, an optimized effective action is obtained for the electromagnetic sector in the lowenergy behavior of the U(1) coupling and the anomalous dimension. In Sec. III, the gravitational sector is taken into account, conducing to a symmetry-breaking potential. Section IV provides expressions for the mass and vacuum expectation value of the Higgs boson as well as a benchmark for gravitational parameters coming from an infrared expansion. Section V connects our results with those obtained in the context of the functional renormalization 
group. In Sec. VI, a discussion of how this new mechanism could shed some light on the problem of quadratic divergences that affects the spin- 0 fields in gauge theories is carried out. Finally, a summary, some comments, and ideas for future work are given in Sec. VII.

\section{Similarities and differences with the Coleman-Weinberg mechanism}

The model presented is strongly reminiscent of the $\mathrm{CW}$ mechanism [65], because neither of the two shows SSB at tree level. However, there are crucial differences, which are explored by outlining both methods.

(i) In the $\mathrm{CW}$ mechanism, the radiative corrections drive the SSB in theories that do not exhibit such breakings at tree level. The theory considers a massless scalar QED where the radiative corrections generate a one-loop effective potential. The mass of the scalar meson is defined as the second derivative of the effective potential evaluated at the arbitrary mass scale $M$, introduced to stay away from the logarithmic singularity in momentum space. The identification of this scale is arbitrary, generically chosen, such that the masses of scalar and vector fields give the observed values. After SSB, the theory can be expressed in terms of one dimensionless $e$ and one dimensionful (the VEV $v$ ). However, some problems with this scale-invariant model still persist. The original prediction for the Higgs mass $(\sim 10 \mathrm{GeV})$ is far from the currently observed value, thus forcing the Higgs self-coupling to be so large that a nearby Landau pole might break the oneloop approximation [66]. Thus, the $\mathrm{CW}$ mechanism necessarily depends on the UV cutoff of the theory to reproduce the current experimental values. Furthermore, if the cutoff scale is of TeV order, the mass of the top quark is less than the observed value $[67,68]$.

(ii) In the present model, we start with a scalar QED theory without quartic interaction, minimally coupled with the metric field. The main consequence of the proposed approach lies in the fact that the oneloop quantum corrections do not generate SSB at the level of the effective action $\Gamma_{k}$. To see the previous statement, consider the one-loop effective potential generated in scalar QED [65]:

$$
\begin{aligned}
V_{\mathrm{eff}}[\phi]= & m^{2} \phi^{2}+\frac{\lambda}{4 !} \phi^{4} \\
& -\frac{1}{4(4 \pi)^{2}}\left\{3\left(2 e^{2} \phi^{2}\right)^{2}\left(\log \frac{M^{2}}{2 e^{2} \phi^{2}}+\frac{3}{2}\right)\right. \\
& +\left(m^{2}+\frac{\lambda \phi^{2}}{4}\right)^{2}\left(\log \frac{M^{2}}{m^{2}+\frac{\lambda \phi}{4}}+\frac{3}{2}\right) \\
& \left.+\left(m^{2}+\frac{\lambda \phi^{2}}{12}\right)^{2}\left(\log \frac{M^{2}}{m^{2}+\frac{\lambda \phi}{12}}+\frac{3}{2}\right)\right\},
\end{aligned}
$$

where $M$ corresponds to an infrared cutoff introduced to avoiding the logarithmic infrared singularity. Since (2.1) does not have quartic interaction, we put $\lambda=0$ in (1.1). The $m$ value can be inferred from the location of the minimum of $V_{\text {eff }}$ at the VEV $\phi=\langle\phi\rangle$. After inserting the coupling $m$ in the effective potential, the coupling $\lambda$ is defined by

$$
\begin{aligned}
\lambda & =\frac{d^{4} V_{\text {eff }}(\langle\phi\rangle)}{d \phi^{4}} \\
& =\frac{27 e^{8}\left(\log \frac{3 e^{2}}{32}-1-2 \log \pi\right)}{1024 \pi^{6}} .
\end{aligned}
$$

In the last step we have used $M=\sqrt{2} \mathrm{e}^{-1 / 4} e\langle\phi\rangle$, with e being the Euler constant. From (1.2) one can deduce that $\lambda$ is proportional to $e^{2}$, i.e., corrections of higher orders. However, by applying the variational parameter setting (2.4), one gets SSB at the level of the optimal effective action $\Gamma_{\text {opt }}$ due to the existence of a vacuum gravitational term, concluding that radiative corrections, together an appropriate way of fixing the renormalization scale, are the dominant driving forces of SSB. Additionally, the unphysical dependence of the renormalization scale is removed from observables. As a consequence, the masses for the scalar and vector fields are functions of the parameters appearing in the original Lagrangian, which include the unknown low-energy expansion of gravitational couplings.

Thus, both approaches have in common the lack of SSB at the level of the classical action and the dependence of physical observables on an arbitrary parameter introduced to avoid infrared logarithmic singularities. However, they differ in the way of generating SSB. In the CW mechanism, radiative corrections are enough to ensure a mass term for the scalar field. In the present model, scalar QED without quartic interaction will be coupled to the gravitational sector, and the symmetry-breaking potential will be driven by the scale-setting condition (2.4). This will allow us to address the dependence on the problematical UV cutoff in physical quantities in a way that is not possible in the $\mathrm{CW}$ formalism.

\section{SCALAR QED WITHOUT QUARTIC INTERACTION}

As a first example, one can consider a theory that contains charged spin-zero particles that interact with photons. The bare action is given by

$$
\begin{aligned}
\mathcal{S}\left(A_{\mu}, \Phi\right)= & \int d^{4} x\left(\frac{a_{b}}{2}\left(D_{\mu} \Phi\right)^{*}\left(D^{\mu} \Phi\right)+\frac{m_{b}^{2}}{2} \Phi^{*} \Phi\right. \\
& \left.-\frac{1}{4 e_{b}^{2}} F_{\mu \nu} F^{\mu \nu}\right),
\end{aligned}
$$


where $D_{\mu}=\partial_{\mu}-i A_{\mu}, F_{\mu \nu}=\partial_{\mu} A_{\nu}-\partial_{\nu} A_{\mu}$, and $\Phi$ is a complex scalar field.

\section{A. Optimized effective action}

Following Wilson's idea [69], one can define an average effective action $\Gamma_{k}$ as the functional obtained after integrating out the quantum fluctuations, which contain momenta $q^{2}>k^{2}$. By changing $k$, this scale-dependent effective action can be seen as a smooth interpolation between the microscopic ultraviolet action $\Gamma_{k \rightarrow \infty}$ and the full quantum effective action in the infrared limit $\Gamma_{k \rightarrow 0}$. The effective action for (2.1) reads

$\Gamma_{k}=\int d^{4} x\left(\frac{a_{k}}{2}\left(D_{\mu} \phi\right)^{*}\left(D^{\mu} \phi\right)+\frac{m_{k}}{2} \phi^{*} \phi-\frac{1}{4 e_{k}^{2}} F_{\mu \nu} F^{\mu \nu}\right)$.

This effective action has no quartic interaction in the scalar field and, therefore, does not show the standard SSB. The couplings $\left(a_{k}, m_{k}, e_{k}\right)$ are now scale-dependent quantities. To avoid the logarithmic divergences appearing in the QED couplings due to deep infrared scale $k \rightarrow 0$, the RG scale is split into its reference fixed part $k_{0}=m_{0}$ and its variable part $k^{\prime}$ as $k=m_{0}+k^{\prime}$. Identifying the reference scale as $m_{0}$ and defining the gravitational couplings in the infrared limit at this scale as shown in Fig. 1, the couplings can be expanded in the vicinity of $m_{0}$ using the dimensionless quantity $\frac{k^{\prime}}{k_{0}}$ as the expansion parameter:

$$
a_{k}=a_{0}+\xi_{a, 1} \frac{k^{\prime}}{m_{0}}+\xi_{a, 2} \frac{k^{\prime 2}}{m_{0}^{2}}+\mathcal{O}\left(\frac{k^{\prime}}{m_{0}}\right)^{3},
$$

$$
\begin{gathered}
m_{k}^{2}=m_{0}^{2}+\xi_{m, 1} \frac{k^{\prime}}{m_{0}}+\xi_{m, 2} \frac{k^{\prime 2}}{m_{0}^{2}}+\mathcal{O}\left(\frac{k^{\prime}}{m_{0}}\right)^{3}, \\
\frac{1}{e_{k}^{2}}=\frac{1}{e_{0}^{2}}+\xi_{e, 1} \frac{k^{\prime}}{m_{0}}+\xi_{e, 2} \frac{k^{\prime 2}}{m_{0}^{2}}+\mathcal{O}\left(\frac{k^{\prime}}{m_{0}}\right)^{3} .
\end{gathered}
$$

The set of coefficients $\left(\xi_{i j}\right)$ with $i=(a, m, e)$ and $j=$ $(1,2)$ is obtained from the beta functions of $(2.1)$. Those beta functions further depend on the renormalization scheme.

It is desirable that physical observables are independent of the particular renormalization scheme used to renormalize the theory and the corresponding unphysical parameters involved in this process. However, if the prediction, calculated by a series of approximations, depends on unphysical parameters, then the parameters should be chosen such that variations will minimize the sensitivity of the observable on those parameters. Following this criterion, one looks for a scale setting of the renormalization scale as a function of physical variables $k=k\left(\phi, \xi_{i}, F_{\mu \nu}, \ldots\right)$. This identification results from applying the variational principle to $k$ by promoting the scale $k$ to a field [23] at the level of the effective action. As shown in Refs. [23,34,70], this minimization can be written as

$$
\begin{aligned}
\frac{\delta \Gamma\left(A_{\mu}, \phi(x), k(x), a_{k}, m_{k}\right)}{\delta k} & =0 \\
\left.\Rightarrow \frac{\mathrm{d} \mathcal{L}\left(A_{\mu}, \phi(x), k(x), a_{k}, m_{k}\right)}{\mathrm{d} k}\right|_{k=k_{\mathrm{opt}}} & =0 .
\end{aligned}
$$

In contrast to most other scale settings, the above procedure allows maintaining the original gauge symmetries (even

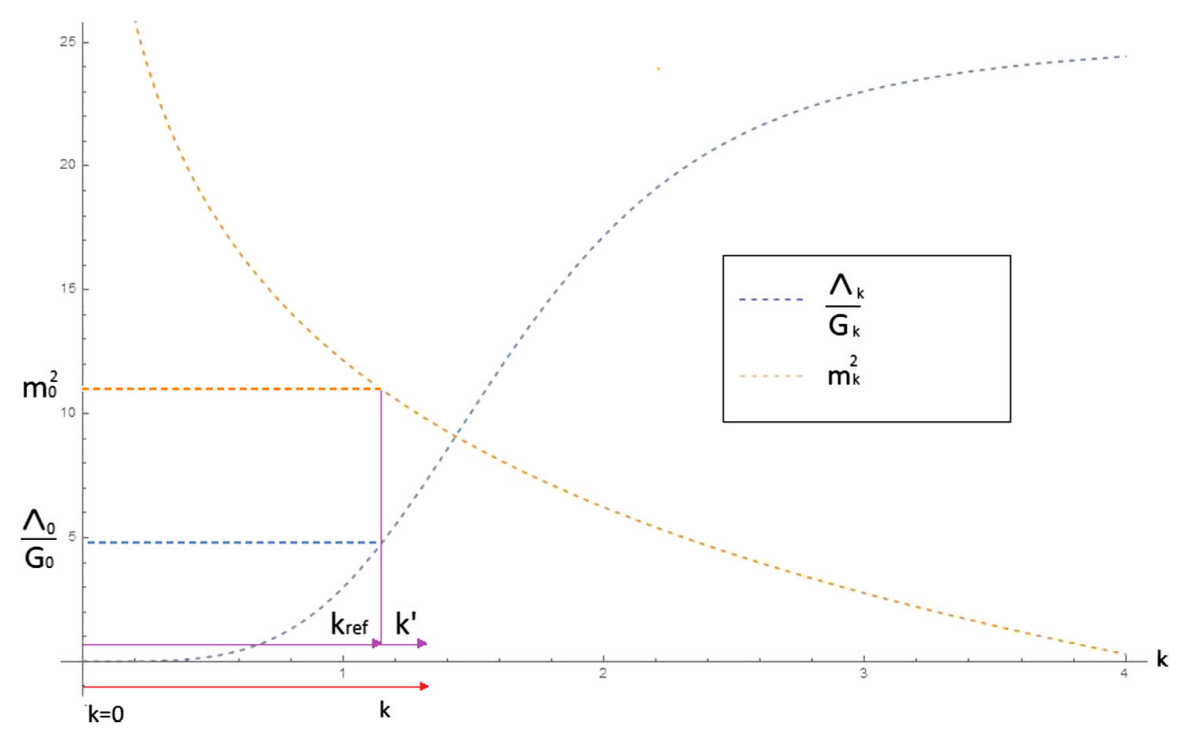

FIG. 1. Graphic representation of the reference scale used in this work. 
diffeomorphism invariance [23]). The philosophy underlying this procedure has been developed in Refs. [23,34,70], and it has been successfully applied in different contexts [71-76]. For consistency, with the expansion (2.3), also the effective action has been expanded up to $k^{2}$. The condition (2.4) allows resolving the renormalization-point ambiguity by selecting a single scale and fixing it as a function of dynamical field variables:

$$
k_{\mathrm{opt}} \rightarrow \frac{-\xi_{e, 1} F_{\mu \nu} F^{\mu \nu}+2 \xi_{a, 1}\left|D_{\mu} \phi\right|^{2}-2 \xi_{m, 1} \phi^{2}}{2\left(\xi_{e, 2} F_{\mu \nu} F^{\mu \nu}-2 \xi_{a, 2}\left|D_{\mu} \phi\right|^{2}+2 \xi_{m, 2} \phi^{2}\right)} .
$$

Since there are no new undetermined integration constants in (2.5), one can insert the solution $k=k_{\text {opt }}$ back into (2.2). This gives an optimal effective action independent of the arbitrary scale $k$ :

$$
\begin{aligned}
\Gamma_{\mathrm{opt}}= & \int d^{4} x\left\{\frac{2 \xi_{e, 1} \xi_{e, 2} \xi_{m, 1}-4 m_{0}^{2} \xi_{e, 2}^{2}-\xi_{e, 1}^{2} \xi_{m, 2}}{8 \xi_{e, 2}^{2}} \phi^{2}\right. \\
& \left.+\frac{\left(\xi_{e, 2} \xi_{m, 1}-\xi_{e, 1} \xi_{m, 2}\right)^{2}}{4 F^{\mu \nu} F_{\mu \nu} \xi_{e, 2}^{3}} \phi^{4}+\mathcal{L}_{\mathrm{kin}}+\mathcal{L}_{\mathrm{const}}+\mathcal{O}\left(\phi^{5}\right)\right\},
\end{aligned}
$$

where the potential has been expanded to order $\phi^{4}$ in a weak field approximation. Kinetic factors of $\phi$ and $A_{\mu}$ are contained in $\mathcal{L}_{\text {kin }}$, and quantities without any field factor $\phi$ are assembled into $\mathcal{L}_{\text {const }}$. One notes that (2.6) has a quadratic and a quartic term in $\phi$, which is necessary for the standard SSB mechanism to take place. It is important to remember that the effective actions [(2.2) and (2.6)] contain already quantum corrections. However, one also notes that the quartic coupling is well behaved only for a finite electromagnetic background field, $\left\langle F_{\mu \nu} F^{\mu \nu}\right\rangle \neq 0$. Even though this is an interesting feature, it is not the type of SSB we are interested in.

\section{B. Values of parameter expansion from QED sector}

It is instructive to calculate the explicit values of the parameters $\xi_{e, j}$ and $\xi_{m, j}$. Those $\xi$ 's have some scheme dependence and can be obtained by applying perturbative methods. When the integral for obtaining an explicit expression of the coupling as a function of the scale is carried out, the lower limit (unlike the case of gravity) cannot be zero, which explains the expansion around $k_{0}$ instead of 0 in (2.3). Expressing $k \frac{\partial \alpha}{\partial k}$, with $\alpha \equiv \frac{e^{2}}{4 \pi}$, in terms of the known scalar QED $\beta$ function up to one loop in the minimal subtraction scheme [77] gives the RG equation

$$
k \frac{\mathrm{d} \alpha}{\mathrm{d} k}=\frac{\alpha^{2}}{6 \pi} .
$$

Integrating this equation between the initial and an intermediate scale, the running coupling takes the form

$$
\alpha\left(k^{2}\right)=\frac{\alpha\left(k_{0}\right)}{1-\frac{1}{3 \pi} \alpha\left(k_{0}\right) \ln \left(\frac{k}{k_{0}}\right)} .
$$

The expansion for the running coupling $e_{k}$ around $k=k_{0}$ by imposing $e_{k} \rightarrow e_{k_{0}} \equiv e_{0}$ for the first term of the series (see Fig. 1) and rearranging (2.8) gives

$\frac{1}{e_{k}^{2}}=\frac{1}{e_{0}^{2}}-\frac{1}{24 \pi^{2}}\left(\frac{k^{\prime}}{m_{0}}\right)+\frac{1}{48 \pi^{2}}\left(\frac{k^{\prime 2}}{m_{0}^{2}}\right)+\mathcal{O}\left(\frac{k^{\prime}}{m_{0}}\right)^{3}$.

The one-loop contribution to the anomalous mass dimension in Lorentz gauge [77], $\gamma_{m}=-\frac{3 e^{2}}{16 \pi^{2}}$, follows the same treatment. Integrating $\gamma_{m}$ with the initial condition in $k_{0}$, an expansion [like (2.9)] for the running coupling $m_{k}$ is obtained:

$$
\begin{aligned}
m_{k}^{2}= & m_{0}^{2}-\frac{3 e_{0}^{2} m_{0}^{2}}{8 \pi^{2}}\left(\frac{k^{\prime}}{m_{0}}\right)+m_{0}^{2} e_{0}^{2}\left(\frac{24 \pi^{2}+e_{0}^{2}(9 \pi-1)}{128 \pi^{3}}\right) \\
& \times\left(\frac{k^{\prime}}{m_{0}}\right)^{2}+\mathcal{O}\left(\frac{k^{\prime}}{m_{0}}\right)^{3} .
\end{aligned}
$$

Thus, the set of parameters $\left(\xi_{i, j}\right)$ from the scalar sector can be identified from a comparison between (2.3), (2.9), and (2.10). One finds

$$
\begin{aligned}
& \xi_{e, 1}=-\frac{1}{24 \pi^{2}}, \\
& \xi_{e, 2}=\frac{1}{48 \pi^{2}}, \\
& \xi_{m, 1}=-\frac{3 e_{0}^{2} m_{0}^{2}}{8 \pi^{2}}, \\
& \xi_{m, 2}=m_{0}^{2} e_{0}^{2}\left(\frac{24 \pi^{2}+e_{0}^{2}(9 \pi-1)}{128 \pi^{3}}\right) .
\end{aligned}
$$

The set of equations (2.11) is valid only for scalar QED up to one loop in perturbation theory in Lorentz gauge and in the minimal subtraction scheme. Possible contributions on these $\mathrm{U}(1)$ coefficients coming from the gravitational sector will be discussed in Sec. IV.

\section{GRAVITATIONAL SECTOR MINIMALLY COUPLED TO A CHARGED SCALAR}

Since the field theoretical content in (2.1) was not providing all ingredients needed for SSB, one needs to take into account some generalization. As explained in the introduction, a cosmological term (vacuum energy density) is important for the implementation of our ideas. Thus, let us consider a gravitational sector coupled to matter. In the leading-order truncation (meaning that higher-order diffeomorphism-invariant operators like $R^{2}, R_{\mu \nu} R^{\mu \nu}, \ldots$ are neglected), the simplest effective action of gravity coupled to a charged scalar reads 


$$
\begin{aligned}
\Gamma_{k}= & \int d^{4} x \sqrt{-g}\left\{\kappa\left(2 \Lambda_{k}-R\right)+\frac{m_{k}^{2}}{2} \phi^{*} \phi\right. \\
& \left.+\frac{a_{k}}{2}\left(D_{\mu} \phi\right)^{*}\left(D^{\mu} \phi\right)-\frac{1}{4 e_{k}^{2}} F^{\mu \nu} F_{\mu \nu}+c_{f} \mathcal{L}_{\mathrm{kin}, \psi}\right\} .
\end{aligned}
$$

One notes that all couplings $\left(\kappa_{k}, \Lambda_{k}, m_{k}, a_{k}, e_{k}\right)$, except the one of the sterile fermions $c_{f}$, are scale-dependent quantities. In this action, $\Lambda_{k}$ stands for the scale-dependent cosmological constant, $R$ is the Ricci scalar, and $\kappa=\left(16 \pi G_{k}\right)^{-1}$, where $G_{k}$ is the running counterpart of the gravitational coupling. In addition to (2.3), an expansion around the infrared zone $k \rightarrow k_{0}$ in the gravitational coupling is needed to get an optimal scale. In order to maintain consistency with the expansion (2.3), the effective action (3.1) and the gravitational couplings also are expanded to the same order. We examine the solutions of the gravitational couplings with an Einstein-Hilbert truncation in the deep infrared. In this limit, one finds, independent of the implementation of the Wilsonian renormalization procedure, a RG running of Newton and the cosmological constant of the form

$$
\begin{gathered}
G(k)=G_{0}\left(1+C_{1} G_{0} k^{2}+C_{2} G_{0}^{2} k^{4}\right)+\mathcal{O}\left(k^{6}\right), \\
\Lambda(k)=\Lambda_{0}+C_{3} \Lambda_{0} G_{0} k^{2}+C_{4} \zeta\left(G_{0} \Lambda_{0}\right) k^{4}+\mathcal{O}\left(k^{6}\right),
\end{gathered}
$$

with $C_{1,2,3,4}$ being real numbers. Depending on the sign of $C_{1}$, Eq. (3.2) shows a screening or antiscreening property of gravity. When an expansion is made around $k_{0}$ instead of zero, one can redefine the constants $C_{i}$, giving

$$
\tilde{\Lambda}_{k}=\tilde{\Lambda}_{0}+\xi_{\tilde{\Lambda}, 1} m_{0}\left(\frac{k^{\prime}}{m_{0}}\right)+\xi_{\tilde{\Lambda}, 2} m_{0}^{2}\left(\frac{k^{\prime}}{m_{0}}\right)^{2}+\mathcal{O}\left(k^{\prime}\right)^{3},
$$

where a change of the cosmological variable $\tilde{\Lambda}_{k}=\frac{\Lambda_{k}}{G_{k}}$ has been applied. Please note that if one is interested in the effective Higgs potential, (3.4) is enough to get an overview of the gravitational contribution to this model. In particular, $G_{k}$ from (3.2) is not needed, because this part of the action is proportional to $R$, which does not take part in the SSB process. Based on a study on field-parametrization dependence of the renormalization group flow in the vicinity of non-Gaussian fixed points in quantum gravity, a beta function derived from EH action can be used to fix the parameters $\xi_{i}$ in (3.4). One can further look at how the massless-matter fields affect asymptotically safe quantum gravity. In this case, the parameters $\xi_{i}$ would have a dependence on the number and the nature of matter fields. For now, the gravitational parameter set $\xi_{\tilde{\Lambda}, j}$ is kept arbitrary. In the next section, a physical benchmark for this set $\xi_{\tilde{\Lambda}, j}$ will be worked out.
As in (2.5), the scale setting is performed by demanding (3.1) to be insensitive under infinitesimal changes of $k$, giving

$k_{\mathrm{opt}}=\frac{\mathcal{H}_{R, 1}+\mathcal{H}_{F, 1}+\mathcal{C}_{1}-2 G_{0}^{3}\left(\xi_{m, 1} \phi^{2}-|D \phi|^{2} \xi_{a, 1}\right)}{\mathcal{H}_{R, 2}+\mathcal{H}_{F, 2}+\mathcal{C}_{2}+4 G_{0}^{3}\left(\xi_{m, 2} \phi^{2}-\xi_{a, 2}|D \phi|^{2}\right)}$.

Herein, the functions $\mathcal{H}_{R}$ and $\mathcal{H}_{F}$ have as arguments the Ricci tensor $R$ and electromagnetic tensor $F_{\mu \nu} F^{\mu \nu}$, respectively, in addition to the infrared value of the running $G_{k}$. The constants $\mathcal{C}_{1,2}$ contain only infrared couplings and electromagnetic constants $\xi_{e, m, \ldots}$. When the optimal scale is inserted back into the effective action (3.1) (that includes gravitational effects), one gets an optimal effective action independent of $k$ :

$$
\begin{aligned}
\Gamma_{\mathrm{opt}}= & \int d^{4} x \sqrt{-g}\left\{+\frac{\mu^{2}}{2}|\phi|^{2}-\frac{\lambda}{4}|\phi|^{4}-\frac{1}{4 \tilde{e}} F_{\mu \nu} F^{\mu \nu}\right. \\
& \left.+\mathcal{L}_{\text {kin }}+\mathcal{L}_{\text {coup }}+\mathcal{L}_{\text {const }}+\mathcal{O}\left(\phi^{6}\right)\right\}
\end{aligned}
$$

where $\mathcal{L}_{\text {kin }}$ contains kinetic terms for the real scalar and gauge fields. Couplings with higher-order factors and Ricci scalar quantities are collected into $\mathcal{L}_{\text {coup }}$, and the Lagrangian part independent of the Ricci scalar, electromagnetic strength, and scalar field is named $\mathcal{L}_{\text {const }}$. The effective potential has again been expanded up to the order of $\phi^{4}$. The optimal effective action (3.6) is written following the usual notation with $\mu$ and $\lambda$ appearing in the Abelian Higgs mechanism:

$$
\begin{aligned}
& \frac{\mu^{2}}{2}=\frac{m_{0}^{2}}{2}+\frac{\xi_{\tilde{\Lambda}, 1}\left(\xi_{m, 2} \xi_{\tilde{\Lambda}, 1}-2 \xi_{m, 1} \xi_{\tilde{\Lambda}, 2}\right)}{8 \xi_{\tilde{\Lambda}, 2}^{2}}, \\
& \frac{\lambda}{4}=\frac{\left(\xi_{m, 2} \xi_{\tilde{\Lambda}, 1}-\xi_{m, 1} \xi_{\tilde{\Lambda}, 2}\right)^{2}}{32 \xi_{\tilde{\Lambda}, 2}^{3}} .
\end{aligned}
$$

If those parameters have the correct values, then the field $\phi$ acquires a VEV, and the U(1) global symmetry will be spontaneously broken. Thus, (3.6) shows that, even if one starts with a model like (3.1), which has no SSB, it is possible to get this feature for the optimal effective action $\Gamma_{\text {opt }}$. This is particularly true if $\mu^{2}, \lambda>0$.

\section{GAUGE BOSON MASSES}

In this section, restrictions on the RG parameters $\xi_{i}$ will be studied. 


\section{A. Mass and vacuum expectation value of scalar and gauge fields}

The parameters $\xi_{m, 1}$ and $\xi_{m, 2}$ appearing in (3.7) can be obtained studying the changes of the anomalous mass dimension. Since the action (3.1) considers the EinsteinHilbert contribution, the gravitational sector may, in principle, have an impact on the behavior of electromagnetic couplings. Gravitational corrections to the beta function in quantum field theories have been analyzed in Refs. [78-87]. Non-Abelian gauge fields coupled to gravity in $(3+1)$ dimensions give rise to an additional term in the one-loop beta function proportional to the inverse square of the Planck scale, improving the asymptotic freedom of $N=4$ super Yang-Mills theory [88,89]. In Ref. [90], it is pointed out that quadratic divergences coming from the gravitational sector are responsible for asymptotic freedom of the QED beta function in a gauge-independent context with an energy scale near the Planck scale. For the case where a complex scalar field is minimally coupling to perturbative quantized Einstein gravity with an explicit gauge dependence in the photon and graviton propagator, the total vacuum polarization tensor depends on the gauge parameters, surface terms, a dimensionless constant, and the ultraviolet momentum cutoff, as explained in Refs. [91,92]. In the last case, there are several reasons for neglecting the gravitational contribution to the usual beta function of the $\mathrm{U}(1)$ gauge coupling:

(i) Choosing the gauge parameter, $\xi$ appearing in the graviton propagator in Ref. [92], equal to $\frac{5}{13}$, a cancellation of a gravitational contribution to be takes place.

(ii) Using dimensional regularization instead of a momentum cutoff, the arbitrary parameter contained in the gravitational term is set to 0 [91].

(iii) Some studies [93-95] have shown the beta function of scalar electrodynamics possesses no contribution coming from gravitational interactions.

(iv) In the infrared $k \approx k_{0}$, all gravitational contributions to the beta functions of matter will be strongly suppressed by the Planck scale. This is the reason why the standard model without gravity is a successful quantum field theory in the first place.
Given the arguments expressed above, the gravitational contribution to the electromagnetic beta functions will be neglected. One condition on the effective potential in (3.6) for producing positive Higgs parameters and then SSB was that $\lambda>0$. This determines the sign of $\xi_{\tilde{\Lambda}, 2}$. This can be seen by replacing (2.11c) and (2.11d) in (3.7b) and demanding $\lambda>0$. For a negative value of $\xi_{\tilde{\Lambda}, 2}$, one has to solve the inequality

$\left[e_{0}^{4}(9 \pi-1) \xi_{\tilde{\Lambda}, 1}+24 e_{0}^{2} \pi^{2}\left(\xi_{\tilde{\Lambda}, 1}+2 m_{0}^{2} \xi_{\tilde{\Lambda}, 2}\right)\right]^{2}<0$,

which has no solution for $e_{0}, \xi_{\tilde{\Lambda}, 1} \in \mathbb{R}$. Thus, the requirement for the field $\phi$ to acquire a VEV is $\xi_{\tilde{\Lambda}, 2}>0$. From (3.6) and (3.7), the VEV of the scalar field is

$\phi_{\mathrm{VEV}}^{2}=\frac{2\left(4 m_{0}^{2} \xi_{\tilde{\Lambda}, 2}^{2}+\xi_{\tilde{\Lambda}, 1}\left(\xi_{m, 2} \xi_{\tilde{\Lambda}, 1}-2 \xi_{m, 1} \xi_{\tilde{\Lambda}, 2}\right)\right) \xi_{\tilde{\Lambda}, 2}}{\left(\xi_{m, 2} \xi_{\tilde{\Lambda}, 1}-\xi_{m, 1} \xi_{\tilde{\Lambda}, 2}\right)^{2}}$.

Suppose that the scalar potential in (3.6) is near one of the minima (say, the positive one); then it is convenient to define a fluctuation of the scalar field $\phi(x)=\phi_{\mathrm{VEV}}+\eta(x)$. The squared mass of the complex scalar field $\eta(x)$ is then

$$
m_{\eta}^{2}=2\left(m_{0}^{2}+\frac{\xi_{\tilde{\Lambda}, 1}\left(\xi_{m, 2} \xi_{\tilde{\Lambda}, 1}-2 \xi_{m, 1} \xi_{\tilde{\Lambda}, 2}\right)}{4 \xi_{\tilde{\Lambda}, 2}^{2}}\right) .
$$

One notes that, unlike in the usual approach (6.1a) and (6.1b), the mass (4.3) does not depend on the gauge couplings and their expansion parameters. Furthermore, the optimal U(1) coupling $\tilde{e}$ in Eq. (3.6) is shifted from its infrared value $e_{0}$ :

$$
\frac{1}{\tilde{e}^{2}}=\frac{1}{e_{0}^{2}}+\frac{\left(4 \xi_{\tilde{\Lambda}, 1}+\xi_{m, 1} \phi_{\mathrm{VEV}}^{2}\right)\left(4 \xi_{e, 2} \xi_{\tilde{\Lambda}, 1}-8 \xi_{e, 1} \xi_{\tilde{\Lambda}, 2}+h \phi^{2}\right)}{4\left(4 \xi_{\tilde{\Lambda}, 2}+\xi_{m, 2} \phi^{2}\right)^{2}},
$$

where $h=\xi_{e, 2} \xi_{m, 1}-2 \xi_{e, 1} \xi_{m, 2}$. The mass term for the gauge bosons is obtained through the product between the inverse of (4.4) and the VEV of the scalar field (4.2):

$$
m_{A}^{2}=\frac{64 \xi_{\tilde{\Lambda}, 2}^{3}\left(\frac{\mathcal{F}_{1}}{4 \xi_{\tilde{\Lambda}}^{2}}-m_{0}^{2}\right)}{\left(\xi_{m, 2} \xi_{\tilde{\Lambda}, 1}-\xi_{m, 1} \xi_{\tilde{\Lambda}, 2}\right)^{2}}\left(\frac{4}{e_{0}^{2}}+\frac{\mathcal{F}_{3}\left(\xi_{e, 2} \mathcal{F}_{3}+6 \xi_{e, 1} \xi_{m, 2} \xi_{\tilde{\Lambda}, 2} \mathcal{F}_{1}-4 \xi_{e, 1} \mathcal{F}_{2}\right)}{\left(3 \xi_{m, 2}^{2} \xi_{\tilde{\Lambda}, 1}^{2} \xi_{\tilde{\Lambda}, 2}-6 \xi_{m, 1} \xi_{m, 2} \xi_{\tilde{\Lambda}, 1} \xi_{\tilde{\Lambda}, 2}^{2}+2 \mathcal{F}_{2}\right)^{2}}\right)^{-1}
$$

with

$$
\begin{aligned}
\mathcal{F}_{1} & =\xi_{\tilde{\Lambda}, 1}\left(2 \xi_{m, 1} \xi_{\tilde{\Lambda}, 2}-\xi_{m, 2} \xi_{\tilde{\Lambda}, 1}\right), \\
\mathcal{F}_{2} & =\xi_{\tilde{\Lambda}, 2}^{3}\left(\xi_{m, 1}^{2}+2 m_{0}^{2} \xi_{m, 2}\right), \\
\mathcal{F}_{3} & =2 \xi_{m, 2}^{2} \xi_{\tilde{\Lambda}, 1}^{3}-3 \xi_{m, 1} \xi_{m, 2} \xi_{\tilde{\Lambda}, 1}^{2} \xi_{\tilde{\Lambda}, 2}+4 m_{0}^{2} \xi_{m, 1} \xi_{\tilde{\Lambda}, 3}^{3} .
\end{aligned}
$$


After insertion of the set of U(1) parameters (2.11) into (4.2) and (4.3), the mass and VEV of the Higgs field are determined as a function of gravitational parameters appearing in the infrared expansion of $\frac{\Lambda_{k}}{G_{k}}$ and $m_{0}$ :

$$
\begin{aligned}
v^{2} & =256 \pi^{3} \xi_{\tilde{\Lambda}, 2}\left(\frac{e_{0}^{4}(9 \pi-1) \xi_{\tilde{\Lambda}, 1}^{2}+512 m_{0}^{2} \pi^{3} \xi_{\tilde{\Lambda}, 2}^{2}+24 e_{0}^{2} \pi^{2} \xi_{\tilde{\Lambda}, 1} \zeta_{1}}{e_{0}^{4}\left(e_{0}^{2}(9 \pi-1) \xi_{\tilde{\Lambda}, 1}+24 \pi^{2} \zeta_{2}\right)^{2}}\right), \\
m_{\eta}^{2} & =\frac{1}{256 \pi^{3} \xi_{\tilde{\Lambda}, 2}^{2}}\left(e_{0}^{4}(9 \pi-1) \xi_{\tilde{\Lambda}, 1}^{2}+512 \pi^{3} m_{0}^{2} \xi_{\tilde{\Lambda}, 2}^{2}+24 e_{0}^{2} \pi^{2} \xi_{\tilde{\Lambda}, 1} \zeta_{1}\right),
\end{aligned}
$$

with $\zeta_{1}=\left(\xi_{\tilde{\Lambda}, 1}+4 m_{0} \xi_{\tilde{\Lambda}, 2}\right)$ and $\zeta_{2}=\left(\xi_{\tilde{\Lambda}, 1}+2 m_{0} \xi_{\tilde{\Lambda}, 2}\right)$. At this point, it is important to make a few comments about the result obtained in (4.7). The initial action (3.6) has no elements to produce mass for the U(1) gauge boson, since the quartic interaction is missing. After applying the VPS procedure, to get an optimal effective action, a symmetrybreaking effective potential appears when the gravitational sector is taken into account. However, the RG expansion parameters, which drive this SSB, must meet certain requirements. The Hermiticity of the optimal Lagrangian implies that the parameters in (3.7) must be real, and, thus, this Lagrangian respects charge, parity, and time-reversal symmetries. Mimicking the usual Higgs mechanism for $\mathrm{SSB}$, the sign of the mass term is chosen negative. Moreover, the effective coupling $\lambda$ must be positive as a requirement for the scalar potential to be bounded from below. As shown above, this implies that $\xi_{\tilde{\Lambda}, 2}>0$.

\section{B. Benchmark of gravitational parameters}

Because of the dependence on infrared coefficients $\xi_{i}$ provided in the expansion of the couplings involved in the theory, one can expect to get restrictions from physically

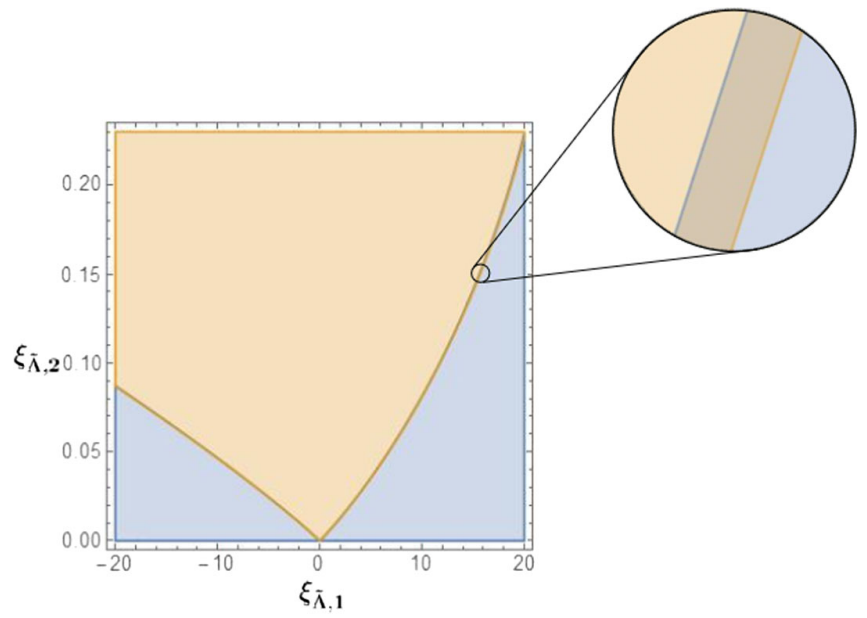

FIG. 2. Allowed parameter range in the gravitational parameter $\xi_{\tilde{\Lambda}, 1}$ and $\xi_{\tilde{\Lambda}, 2}$. The orange region represents gravitational parameters which give a mass for the $\mathrm{U}(1)$ gauge boson lower than $m_{\eta}=125.33 \mathrm{GeV}$, while the blue region represents parameters greater than $m_{\eta}=124.85$ (lower and upper experimental limit of the mass of the Higgs boson). observed gauge boson masses. This exercise is done despite the fact that the model presented in (3.6) is more a conceptual case study rather than a competitive phenomenological model, since it has neither electroweak nor Yukawa couplings implemented. Observed experimental values of the Higgs mass and the VEV will be employed to get a better idea about the distribution of the allowed parameters $\xi_{\tilde{\Lambda}, 1}$ and $\xi_{\tilde{\Lambda}, 2}$ in agreement with the observations. The quantities $m_{\eta}$ and $v$ in (4.7) have four free parameters: $e_{0}, m_{0}, \xi_{\tilde{\Lambda}, 1}$, and $\xi_{\tilde{\Lambda}, 2}$. We impose that

(i) $e_{0}=\sqrt{4 \pi \alpha} \approx 0.3028$ [96]. - The $\mathrm{U}(1)$ coupling $e_{0}$ in (3.1) takes the value of the vertex function in spinor electrodynamics when all three particles (one incoming fermion, incoming photon, and outgoing fermion) are on shell, i.e., the elementary charge $e$. In the deep infrared, the choice $k^{\prime} \rightarrow 0$ is justified due to the long-range character of QED. One confirms numerically that the difference between $\tilde{e}$ and $e_{0}$ is negligible.

(ii) $v=\left(\sqrt{2} G_{F}\right)^{-1 / 2}=246.2197 \mathrm{GeV}$ [97]—Because the experimental uncertainty on the Higgs mass $m_{H}$ is much larger than the uncertainty on the VEV of the Higgs field measured in the muon decay $v_{H}$, only the best fit value $v=246.2197$ will be considered to fix $m_{0}$ as a function of the gravitational parameters $\xi_{\tilde{\Lambda}, 1}$ and $\xi_{\tilde{\Lambda}, 2}$.

(ii) $\xi_{\tilde{\Lambda}, 2}>0$. - When the two preceding points are applied to $(4.7 \mathrm{~b})$, the bound on $\xi_{\tilde{\Lambda}, 2}$ arises from imposing real values for $m_{\eta}$.

The boundaries for the gravitational parameters $\xi_{\tilde{\Lambda}, 1}$ and $\xi_{\tilde{\Lambda}, 2}$ can be obtained by associating the limits of the Higgs boson mass with the limits of $m_{\eta}$ in (4.7b). The result is shown in Fig. 2. Parameters enclosed in the shadow region meet the experimental requirements previously discussed.

\section{COMPARISON WITH THE FUNCTIONAL RENORMALIZATION GROUP}

Up to now, our calculations never made use of a specific shape of gravitational beta functions. Let us make up leeway. The evolution of the scale-dependent dimensionless couplings is dictated by the functional renormalization group equation (also known as the Wetterich equation) 


$$
\frac{\mathrm{d} \Gamma_{k}}{\mathrm{~d} t}=\frac{1}{2} \mathrm{~S} \operatorname{Tr}\left(\frac{\partial_{t} \mathcal{R}_{k}}{\Gamma_{k}^{(2)}[\phi]+\mathcal{R}_{k}}\right)
$$

The Wetterich equation is formulated such that it depends on renormalization group time $t=\ln \frac{k}{k_{0}}$, the modified inverse propagator $\Gamma_{k}^{(2)}[\phi]+\mathcal{R}_{k}$ involving a second functional derivative of the scale-dependent effective action with respect to the fields, and the momentum cutoff $\mathcal{R}_{k}$. The cutoff is chosen such that it suppresses the contributions of field modes smaller than the cutoff scale $k^{2}$ [29,98-100]. The notation STr stands for "supertrace," which is performed over momenta as well as particle species and spacetime or internal indices, including a factor $(-1)$ for fermionic fields. Despite the fact that the Wetterich equation (5.1) is an exact one-loop equation, for practical computations, one has to apply truncations in order to obtain a manageable theory space. The ground for the gravity-matter beta functions used in this section was laid in Refs. [61-64].

To perform the comparison between (3.2), (3.7), and the functional renormalization group, Eq. (5.1), a matter sector is required in addition to the usual Einstein-Hilbert truncation. The matter content consists of $N_{s}$ scalar fields $\phi^{i}$, $N_{D}$ fermion fields $\psi^{i}$, and $N_{V}$ Abelian gauge fields $A_{\mu}^{i}$. In addition to this, one has the ghost and antighost fields $c$ and $\bar{c}$, respectively, all coupled to an external metric $g_{\mu \nu}$. The matter part of the action is given by

$$
\begin{aligned}
& \Gamma_{\text {matter }}=S_{S}(\phi, g)+S_{D}(\psi, \bar{\psi}, g)+S_{V}(A, c, \bar{c}, g), \\
& S_{S}(\phi, g)= \frac{1}{2} \int d^{d} x \sqrt{g} g^{\mu \nu} \sum_{i}^{N_{S}} \partial_{\mu} \phi^{i} \partial_{\nu} \phi^{i}, \\
& S_{D}(A, c, \bar{c}, g)=i \int d^{d} x \sqrt{g} \sum_{i}^{N_{D}} \bar{\psi}_{i} \not D \psi^{i}, \\
& S_{V}(A, c, \bar{c}, g)= \frac{1}{4} \int d^{d} x \sqrt{g} \sum_{i=1}^{N_{V}} g^{\mu \nu} g^{\kappa \lambda} F_{\mu \kappa}^{i} F_{\nu \lambda}^{i} \\
&+\frac{1}{2 \xi} \int d^{d} x \sqrt{g} \sum_{i=1}^{N_{V}}\left(g^{\mu \nu} \nabla_{\mu} A_{\nu}^{i}\right)^{2} \\
&+\int d^{d} x \sqrt{g} \sum_{i=1}^{N_{V}} \bar{c}_{i}\left(-\nabla^{2}\right) c_{i},
\end{aligned}
$$

where $\not D=\gamma^{a} e_{a}^{\mu} \nabla_{\mu}$, with the orthonormal frame $e_{a}^{\mu}$ and where $i$ is a summation index over matter species. Adding to this action the contribution of the Einstein Hilbert truncation (using a type-II cutoff), ghost and gauge fixing, the beta functions in four dimensions for the gravitational scale-dependent coupling are

$$
\begin{aligned}
\beta_{g}= & 2 g+\frac{g^{2}}{6 \pi}\left(N_{S}+2 N_{D}-4 N_{V}-46\right), \\
\beta_{\lambda}= & -2 \lambda+\frac{g}{4 \pi}\left(N_{S}-4 N_{D}+2 N_{V}+2\right) \\
& +\frac{g \lambda}{6 \pi}\left(N_{S}+2 N_{D}-4 N_{V}-16\right) .
\end{aligned}
$$

Here, (5.7) was expanded up to second order in $g$ and $\lambda$. The numbers $-46,+2$, and -16 describe the contribution of the ghost and metric sector, and they depend on the type of cutoff $\mathcal{R}_{k}$ which is used.

In the following analysis, the results from Sec. III will be compared with the evolution of gravitational couplings using the functional renormalization group equation (FGRE). To find suitable conditions for the relevant variables involved in the comparison, one shall vary the numbers of fields $N_{D}, N_{S}$, and $N_{V}$ such that the conditions encountered in the AS scenario are met. The flow equations (5.7a) and (5.7b) can be integrated analytically, and the dimensionful running version of Newton coupling turns out to be

$$
G_{N}=\frac{G_{k}}{1+\frac{1}{2} \mathcal{C}_{1} k^{2} G_{k}}
$$

where $G_{N}$ is the Newton constant measured in the deep infrared $k \rightarrow 0$. The dimensionful running version of the cosmological constant gives

$$
\begin{aligned}
\Lambda_{k}= & -\frac{\mathcal{C}_{2}}{\mathcal{C}_{3}\left(\mathcal{C}_{1}+\mathcal{C}_{3}\right)}\left(\frac{2+\left(\mathcal{C}_{1}+\mathcal{C}_{3}\right) k^{2} G_{k}}{G_{k}}\right) \\
& +\frac{\Lambda_{0} G_{N}\left(2+\mathcal{C}_{1} k^{2} G_{k}\right)^{1+\left(\mathcal{C}_{1} / C_{3}\right)}}{2^{1+\left(\mathcal{C}_{3} / \mathcal{C}_{1}\right)} G_{k}} \\
& +\frac{2^{-\mathcal{C}_{3} / \mathcal{C}_{1}} \mathcal{C}_{2}\left(2+\mathcal{C}_{1} k^{2} G_{k}\right)^{1+\left(\mathcal{C}_{3} / \mathcal{C}_{1}\right)}}{\mathcal{C}_{3}\left(\mathcal{C}_{1}+\mathcal{C}_{3}\right) G_{k}} .
\end{aligned}
$$

In (5.8) and (5.9), we have defined

$$
\begin{aligned}
& \mathcal{C}_{1}=\frac{1}{6 \pi}\left(N_{S}+2 N_{D}-4 N_{V}-46\right), \\
& \mathcal{C}_{2}=\frac{1}{4 \pi}\left(N_{S}-4 N_{D}+2 N_{V}+2\right), \\
& \mathcal{C}_{3}=\frac{1}{6 \pi}\left(N_{S}+2 N_{D}-4 N_{V}-16\right) .
\end{aligned}
$$

Consider the expansion around the quantity $k=\left(k^{\prime}+\right.$ $\left.m_{0}\right) / m_{0}$ (see Fig. 1). In the infrared, the modified running $\tilde{\Lambda}$ up to order $k^{\prime 2}$ reads

$$
\begin{aligned}
\tilde{\Lambda}_{k}= & \tilde{\Lambda}_{0}+m_{0}^{4} \mathcal{C}_{2} \cdot\left(\frac{k^{\prime}}{m_{0}}\right)+3 \cdot 2^{-\mathcal{C}_{1}} m_{0}^{4} \mathcal{C}_{2} \cdot\left(\frac{k^{\prime}}{m_{0}}\right)^{2} \\
& +\mathcal{O}\left(G_{0}, \Lambda_{0}\right) .
\end{aligned}
$$




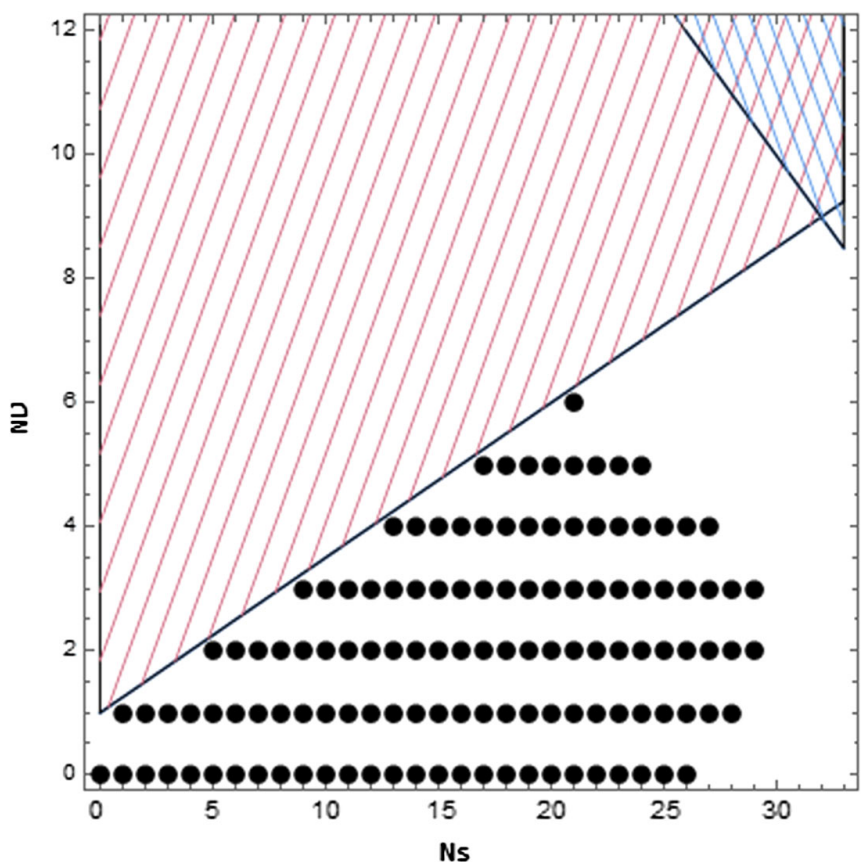

FIG. 3. Dynamical matter degrees of freedom compatible with a gravitational fixed point with two relevant directions for $N_{V}=1$ (explicit values are listed in Appendix A), represented by black bullets. The shaded blue region represents a zone where a negative Newton fixed point takes place, while the shaded red area contains points associated with $\xi_{\tilde{\Lambda}, 2}<0$.

Since the crucial requirement of the method described in the two previous sections was $\xi_{\tilde{\Lambda}, 2}>0$, the compatibility will be dictated by the sign of $\mathcal{C}_{2}$. To compare our result with those found in the AS program, the requirements that the gravitational parameters $\left(\xi_{\tilde{\Lambda}, 1}, \xi_{\tilde{\Lambda}, 2}\right)$ need to meet can be summarized as follows.

(1) Positive Newton fixed point $g^{*}>0$. - The low value $\left(k \lesssim M_{\mathrm{pl}}\right.$,) of Newton gravitational coupling is restricted by observations based on laboratory experiments at the scale $k_{\text {lab }} \simeq 10^{-5} \mathrm{eV}$.

(2) Relevant directions.- - Insofar as the corresponding fixed points for the gravitational couplings of a pure gravity theory have two relevant directions [61], one expects that the addition of a small number of matter degrees of freedom does not change this behavior and the subsequent parametrization in theory space.

(3) Positive value of $\xi_{\tilde{\Lambda}, 2}>0$.-As discussed before, the requirement to ensure that our model guarantees SSB at the level of the effective action needs $\lambda>0$ in (3.7) or, equivalently, $\mathcal{C}_{2}>0$ in (5.11).

The first two criteria were already pointed out in Ref. [61], and they are shown in Appendix A for different matter field configurations, while the third selection criterium is necessary for the validity in (3.7). These conditions determine how many fields $N_{S}, N_{D}, \ldots$ may be incorporated such that the proposed mechanism for SSB is in agreement with the requests of AS. Figure 3 shows how the conditions above allow one to put restrictions on the field content in the $N_{S}-N_{D}$ plane for $N_{V}=1$. Each black point indicates a viable model with SSB and an UV fixed point.

\section{APPLICATION TO TECHNICAL NATURALNESS AND HIERARCHY PROBLEM}

The measurements of the Higgs boson at the Large Hadron Collider [101,102] confirms that its existence and properties are consistent with the SM. Unlike all other particles of the SM, the Higgs boson is a fundamental scalar, which gives rise to the question whether the SSB mechanism, which is induced by the Higgs field, is natural [103-118]. The central issue is the strong sensitivity of masses of scalar particles under radiative corrections, leading to the so-called hierarchy problem and the failure of the notion of naturalness. It is, of course, possible that naturalness is not always a good guiding principle for the understanding of nature [119], but if one would have the choice, a natural description is certainly preferable.

The hierarchy problem affects only scalar particles, since Dirac and gauge fields are technically natural. In the SM, the term $m^{2} H^{\dagger} H$, with $H$ being the $\mathrm{SU}(2)_{L}$ Higgs field doublet, is invariant under any gauge or global symmetry acting on it. If the theory under consideration possesses multiple mass scales, one finds that the Higgs mass $m$ accumulates quantum corrections from all (coupled) particles at all energy scales. Thus, $m$ is affected by heavy particles through the appearance of quadratic divergences (unlike the technically natural spin- $-\frac{1}{2}$ or spin-1 fields). Specifically, in an effective field theory approach of the SM, where the momenta of virtual particles are involved, the radiative corrections are cut off at the scale $\Pi$. The dependence of $m$ on some experimental scale $\mu$ can be obtained from Ref. [120]:

$$
\begin{aligned}
m_{H}^{2}(\mu) & =m_{0}^{2}(\Pi)+\delta m^{2}, \\
\delta m^{2} & =\frac{\Pi^{2}}{16 \pi}\left(\frac{3}{4} g_{1}^{2}+\frac{9}{4} g_{2}^{2}+3 \lambda_{h}^{2}-12 \lambda_{t}^{2}\right),
\end{aligned}
$$

where $\lambda_{h}, \lambda_{t}, g_{1}$, and $g_{2}$ are the Higgs quartic, Yukawa, electroweak $\mathrm{SU}(2)_{L}$, and hypercharge couplings, respectively. Following Eq. (6.1a), an explanation of why the observed Higgs mass remains small requires a large finetuning such that the tree-level parameter exactly cancels the huge correction in Eq. (6.1b). If one starts with a bare action, the quadratic divergences for scalar fields always arise when the quantum corrections are incorporated. Accordingly, a light Higgs scalar cannot survive in a natural way if the theory is expected to hold up to large energy scales, such as the Planck scale. This is referred to as the fine-tuning, hierarchy, or naturalness problem and turns 
out to be independent of the scheme one uses to renormalize the theory.

The new method of SSB introduced in Sec. III allows addressing the problem of quadratic divergences coming from the scalar sector. The bare action $S(\phi)$ is chosen such that no quadratic divergences appear when quantum corrections are incorporated in the effective action $\Gamma_{k}(\Phi)$ [Eq. (3.1)]. Breaking of U(1) symmetry, together with the corresponding masses for scalar and gauge fields, takes place only at $\Gamma_{\text {opt }}$ (3.6) (after having fixed the renormalization scale $k$ ). The advantage of this is those quadratic divergences arising from quantum corrections of the bare action $S$ in the standard SSB are absent when SSB occurs only at the level of $\Gamma_{\mathrm{opt}}$, since the optimal effective action has all quantum corrections already incorporated [38,39]. No additional quantum corrections have to be incorporated into $\Gamma_{\mathrm{opt}}$, and, thus, no quadratic divergences occur.

There exist different notions of naturalness in the context of Higgs and gauge boson masses. It is thus important to clarify in which sense the results for the gauge and scalar masses (4.5), (4.7b) are natural and in which sense the applicability of the method is limited.

(i) The first observation is that the masses do not depend on the UV cuts of the theory, which is necessary for naturality.

(ii) The second observation is that the masses depend, apart from the RG parameters $\xi$, only on the IR parameter $m_{0}$ of the scalar sector, which suggests that the method is insensible to UV contributions from the scalar sector.

(iii) UV contributions from gravitational physics could destroy naturalness, if a dimensionful UV parameter of the model, such as the Planck mass $\left(1 / \sqrt{G_{0}}\right)$, would appear in Eq. (4.5) or (4.7b). This is not the case, which means that this condition for naturalness also holds.

(iv) There could exist a very heavy fermion with a mass $m_{f} \gg m_{0}$ or additional dimensionful gravitational couplings which are not part of this model. These couplings would then appear in the beta functions of the model and, thus, potentially also in the masses (4.5) or (4.7b). This has to be checked model by model and coupling by coupling. It is known that the existence of new heavy particles (either boson or fermion) does not necessarily violate our current naturalness notion, as discussed in Ref. [2]. The large cancellations occurring in the mass squared parameter depend on the nature of the new heavy states, and many theoretical models like the seesaw theory of neutrino masses [121-123], electroweakcoupled fermions as weakly interacting massive particle candidates for dark matter [124], and Higgs portal dark matter [125] meet the naturalness criteria $\delta \mu^{2} \lesssim 100^{2} \mathrm{GeV}^{2}$. (v) There is also the possibility that there are no new states between the Higgs mass and the Planck scale. Such scenarios are known as "desert" scenarios $[126,127]$. In such a scenario, no additional states would threaten the naturalness of a self-consistent model, like the one presented here.

Thus, one can conclude that the presented model produces natural gauge boson and scalar masses but that this statement is limited to the field and couplings which are part of the model.

\section{SUMMARY AND CONCLUSION}

In the present work, a novel mechanism for spontaneous symmetry breaking is suggested that circumvents the appearance of quadratic divergences by avoiding the breaking from taking place at the classical level. It is shown that SSB still can occur at the quantum level, namely, after setting the renormalization scale $k$ of the effective quantum action $\Gamma_{k}$. As a scale-setting procedure, the VPS method is used. This allows arriving at an optimized effective quantum action $\Gamma_{\text {opt }}$, which, under certain conditions, produces SSB. It is intriguing that this procedure even works in flat background spacetime. The naturalness of the resulting Higgs mass is limited to the particle content of the model and possible extensions and modifications have to be studied case by case.

Despite the fact that the toy models of our study do not contain Yukawa, weak, or strong couplings, the underlying mechanism can be expected to work also for models containing these features. It is shown that, within this type of model, one can impose phenomenological conditions on $\Gamma_{\text {opt }}$. These conditions (in particular, the requirement $\xi_{\tilde{\Lambda}, 2}>0$ ) do then allow one to put restrictions on the free parameters and the number of scalar, vector, and Dirac fields. For the example of asymptotically safe quantum gravity coupled to matter, it is shown that, for a given number of scalar fields, these conditions impose an upper and a lower bound on the number of Dirac fields, as shown in Fig. 3.

We further analyze to which extent the results depend on the gauge choice, the truncation, and the shape of the cutoff function in Appendix B. The inclusion of a graviton and ghost anomalous dimension, as well as the anomalous dimension of the matter fields, derived in Ref. [61], does not affect the findings discussed above.

In a future study, we plan to perform an implementation with all couplings necessary to arrive at the GlashowWeinberg-Salam model [128-130] coupled to gravity.

\section{ACKNOWLEDGMENTS}

The work of B. K. was supported by Fondecyt 1181694. 


\section{APPENDIX A: FIXED POINTS AND RELEVANT DIRECTIONS}

TABLE I. Selected gravitational fixed points and relevant directions for $N_{V}=1$ for type-II cutoff, Feynman-de Donder gauge, and one-loop approximation. The first and second columns indicate the matter content. The third and fourth columns are the fixed points for the Newton and cosmological constant. The fifth and sixth columns represents the negative value of the critical exponents.

\begin{tabular}{lccccc}
\hline \hline$N_{s}$ & $N_{D}$ & $g^{*}$ & $\lambda^{*}$ & $\theta_{1}$ & $\theta_{2}$ \\
\hline 0 & 1 & 0.7891 & -0.0355 & 3.4679 & 1.8295 \\
4 & 1 & 0.7874 & 0.0474 & 3.3244 & 1.9828 \\
8 & 1 & 0.7712 & 0.1323 & 3.3392 & 2.1429 \\
12 & 1 & 0.7985 & 0.2119 & 4.7225 & 1.6221 \\
16 & 1 & 1.984 & 0.236 & 16.0381 & 4.2369 \\
20 & 1 & 5.8534 & 0.1827 & 59.1008 & 15.8484 \\
25 & 1 & 27.9783 & 0.0831 & 468.104 & 56.6011 \\
30 & 1 & -7.4924 & -3.0458 & 4.3406 & 3.0051 \\
35 & 1 & -4.3416 & -2.2382 & 4.2789 & 2.7502 \\
0 & 2 & 0.9061 & -0.1388 & 3.6279 & 1.6733 \\
4 & 2 & 0.9255 & -0.0513 & 3.5503 & 1.7274 \\
8 & 2 & 0.9161 & 0.0457 & 3.5004 & 1.8615 \\
12 & 2 & 0.8959 & 0.1407 & 3.8755 & 1.9107 \\
16 & 2 & 1.0522 & 0.2183 & 7.004 & 1.1397 \\
20 & 2 & 2.9317 & 0.2021 & 23.4523 & 5.4593 \\
25 & 2 & 13.2672 & 0.0905 & 139.134 & 16.5161 \\
30 & 2 & -6.5262 & -2.1898 & 4.6413 & 2.9661 \\
0 & 4 & 1.2332 & -0.4412 & 3.8211 & 1.4862 \\
4 & 4 & 1.3634 & -0.3722 & 3.8152 & 1.4125 \\
8 & 4 & 1.4801 & -0.2736 & 3.8299 & 1.3405 \\
12 & 4 & 1.5275 & -0.1386 & 3.9264 & 1.3165 \\
16 & 4 & 1.4747 & 0.0126 & 4.1321 & 1.6424 \\
20 & 4 & 1.5462 & 0.1276 & 6.4885 & 1.4076 \\
25 & 4 & 4.5202 & 0.0938 & 24.9725 & 2.0416 \\
0 & 6 & 1.7796 & -0.9772 & 3.919 & 1.3963 \\
4 & 6 & 2.2679 & -1.073 & 3.9343 & 1.2565 \\
8 & 6 & 3.2689 & -1.3068 & 3.9642 & 1.0238 \\
12 & 6 & 10.5941 & -3.5853 & 3.9829 & 0.3888 \\
0 & 8 & 2.8677 & -2.096 & 3.9688 & 1.3603 \\
4 & 8 & 5.0095 & -3.304 & 3.984 & 1.1922 \\
8 & 8 & 63.0118 & -112.51 & 2.1179 & -1.0674 \\
0 & 9 & 3.9567 & -3.2412 & 3.9837 & 1.3547 \\
2 & 9 & 5.6446 & -4.423 & 3.99 & 1.2799 \\
4 & 9 & 10.5569 & -7.9074 & 3.9964 & 1.1821 \\
6 & 9 & $3.72 \times 10^{7}$ & -1087.061 & $1.25 \times 10^{9}$ & $6.22 \times 10^{8}$ \\
0 & 10 & 6.1542 & -5.5762 & 3.9945 & 1.3547 \\
2 & 10 & 11.888 & -10.3525 & 4 & 1.2801 \\
4 & 10 & 712554.8395 & 490.2162 & $3.487 \times 10^{6}$ & $1.74 \times 10^{6}$ \\
0 & 11 & 12.9664 & -12.8612 & 4.0024 & 1.3589 \\
\hline \hline & & & & &
\end{tabular}

\section{APPENDIX B: CONSISTENCY OF FLOW EQUATIONS}

Formulations of the FRGE require the inclusion of an IR regulator to ensure the integration of all degrees of freedom of fields possessing fluctuations of momenta higher than $k$. The choice of the arguments of the cutoff function $\mathcal{R}_{k}$ gives rise to diversity in the shape of the couplings' flow involved in the theory. However, physical results must remain independent of the selection of the shape and the corresponding endomorphism used in the cutoff function. Thus, one has to check whether the results obtained in this work are independent of this choice. Since the criteria for discriminating the result of the presented mechanism have to do with the sign of $\mathcal{C} 2$, different truncations with various types of cutoff and expansion of the cosmological constant were investigated, setting $N_{S}=2, N_{V}=1$, and the number of Dirac fields being a number between 2 and 9, where we know the model works while fulfilling the conditions required by the AS. The results presented in Table II confirm that this analysis is robust under changes in the truncation procedure.

Note that the characterization of $\mathcal{C}_{2}$ is robust under changes of the gauge choice [133]. Because of the structure of the $\mathcal{C}_{2}$ term in $(5.10 \mathrm{~b})$, the sign of $\xi_{\tilde{\Lambda}, 2}$ is also independent of how many scalar fields are incorporated while keeping $N_{D}$ at some fixed number.

Most of the findings presented in this Appendix might seem evident, but some issues appear when one gets solutions of the FRGE with one or the other of the kinetic operator. In particular, Refs. [132,134,135] show that the spectrum of $\nabla^{2}$ (type-I cutoff) and $\nabla^{2}-\frac{R}{4}$ (type-II cutoff) may turn out in ambiguities in the sign of the fermionic contribution to the running of the Newton constant. In other words, the background-field dependence of $\mathcal{R}_{k}$ can alter results in the background approximation of physical observables, since only a type-II cutoff gives the sign according to the infrared observation of $G$. This result has been corroborated by employing a completely independent method to evaluate the rhs of Eq. (5.1) [135]. However, the SSB discussed in this paper takes place independent of this sign and independent of the gauge choices. 
TABLE II. The compatibility of the result obtained in (3.6) is investigated for various studies of the gravitational RG flow in the presence of SM massless matter fields minimally coupled to an external metric. In the third column, different choices of the gauge parameters $\alpha$ and $\beta$ are explored. The information exhibited in the column "specifies" contains the choice of the covariant differential operator used as the argument of the cutoff function and the expansion of the cosmological constant, explained in the references of the first column. The fifth column gives the sign of the relevant parameter involved in the process of SSB in Eq. (3.7).

\begin{tabular}{|c|c|c|c|c|}
\hline Ref. & Truncation & Gauge & Specifics & $\operatorname{sgn}\left(\xi_{\tilde{\Lambda}, 2}\right)$ \\
\hline [61] & EH with SM matter & $\alpha=\beta=1$ & Type-Ia cutoff lowest order in $\Lambda$ & Positive \\
\hline [61] & EH with SM matter & $\alpha=\beta=1$ & Type-Ia cutoff first order in $\Lambda$ & Positive \\
\hline [61] & EH with SM matter & $\alpha=\beta=1$ & Type-Ib cutoff lowest order in $\Lambda$ & Positive \\
\hline [61] & EH with SM matter & $\alpha=\beta=1$ & Type-Ib cutoff first order in $\Lambda$ & Positive \\
\hline [61] & EH with SM matter & $\alpha=\beta=1$ & Type-II cutoff lowest order in $\Lambda$ & Positive \\
\hline$[61]$ & EH with SM matter & $\alpha=\beta=1$ & Type-II cutoff first order in $\Lambda$ & Positive \\
\hline [131] & EH with SM matter & $\alpha=0, \beta=1$ & Type-II cutoff lowest order in $\Lambda$ & Positive \\
\hline [131] & EH with SM matter & $\alpha=0, \beta=1$ & Type-II cutoff first order in $\Lambda$ & Positive \\
\hline [132] & $f(R)$ to $R^{9}$ with SM matter & $\alpha=0, \beta=-\infty$ & Type-I cutoff lower order in $\Lambda$ & Positive \\
\hline [132] & $f(R)$ to $R^{9}$ with SM matter & $\alpha=0, \beta=-\infty$ & Type-I cutoff first order in $\Lambda$ & Positive \\
\hline [132] & $f(R)$ to $R^{9}$ with SM matter & $\alpha=0, \beta=-\infty$ & Type-II cutoff lower order in $\Lambda$ & Positive \\
\hline [132] & $f(R)$ to $R^{9}$ with SM matter & $\alpha=0, \beta=-\infty$ & Type-II cutoff first order in $\Lambda$ & Positive \\
\hline
\end{tabular}

[1] G. 't Hooft, NATO Sci. Ser. B 59, 135 (1980).

[2] A. de Gouvea, D. Hernandez, and T. M. P. Tait, Phys. Rev. D 89, 115005 (2014).

[3] H. Georgi, H. R. Quinn, and S. Weinberg, Phys. Rev. Lett. 33, 451 (1974).

[4] L. Susskind, Phys. Rev. D 20, 2619 (1979).

[5] M. Dine, Annu. Rev. Nucl. Part. Sci. 65, 43 (2015).

[6] G. F. Giudice, Naturally speaking: The naturalness criterion and physics at the LHC, in Perspectives on LHC Physics, edited by G. Kane and A. Pierce, pp. 155-178 [arXiv:0801.2562].

[7] L. D. Landau, E. M. Lifshitz, J. B. Sykes, J. S. Bell, and E. H. Dill, Phys. Today 14, No. 10, 48 (1961).

[8] L. E. Sadler, J. M. Higbie, S. R. Lerlie, M. Vengalattore, and D. M. Stamper-Kurn, Nature (London) 443, 312 (2006).

[9] M. G. Velarde and C. Normand, Sci. Am. 243, 92 (1980).

[10] L. Sneddon, Phys. Rev. A 24, 1629 (1981).

[11] V. L. Ginzburg and L. D. Landau, Zh. Eksp. Teor. Fiz. 20, 1064 (1950).

[12] S. Weinberg, Prog. Theor. Phys. Suppl. 86, 43 (1986).

[13] F. Englert and R. Brout, Phys. Rev. Lett. 13, 321 (1964).

[14] P. W. Higgs, Phys. Rev. Lett. 13, 508 (1964).

[15] G. S. Guralnik, C. R. Hagen, and T. W. B. Kibble, Phys. Rev. Lett. 13, 585 (1964).

[16] J. D. Bekenstein, Found. Phys. 16, 409 (1986).

[17] P. Moniz, P. Crawford, and A. Barroso, Classical Quant. Grav. 7, L143 (1990).

[18] K. Krasnov, Phys. Rev. D 85, 125023 (2012).

[19] S. Alexander, A. Marciano, and L. Smolin, Phys. Rev. D 89, 065017 (2014).

[20] M. Rinaldi and L. Vanzo, Phys. Rev. D 94, 024009 (2016).

[21] S. Alexander, J. D. Barrow, and J. Magueijo, Classical Quant. Grav. 33, 14LT01 (2016).
[22] E. Guendelman, E. Nissimov, and S. Pacheva, Int. J. Mod. Phys. D 25, 1644008 (2016).

[23] B. Koch, P. Rioseco, and C. Contreras, Phys. Rev. D 91, 025009 (2015).

[24] P. M. Stevenson, Phys. Rev. D 23, 2916 (1981).

[25] J. Avan and H. J. de Vega, Phys. Rev. D 29, 2891 (1984).

[26] E. A. Uehling, Phys. Rev. 48, 55 (1935).

[27] W. Dittrich and M. Reuter, Lect. Notes Phys. 220, 1 (1985).

[28] A. Bonanno and M. Reuter, Phys. Rev. D 60, 084011 (1999).

[29] C. Wetterich, Phys. Lett. B 301, 90 (1993).

[30] M. Reuter and C. Wetterich, Nucl. Phys. B417, 181 (1994).

[31] M. Reuter and C. Wetterich, Nucl. Phys. B391, 147 (1993).

[32] A. Bonanno and M. Reuter, Phys. Rev. D 62, 043008 (2000).

[33] D. F. Litim and T. Plehn, Phys. Rev. Lett. 100, 131301 (2008).

[34] B. Koch and I. Ramirez, Classical Quant. Grav. 28, 055008 (2011).

[35] C. Contreras, B. Koch, and P. Rioseco, Classical Quant. Grav. 30, 175009 (2013).

[36] B. Koch and F. Saueressig, Classical Quant. Grav. 31, 015006 (2014).

[37] C. Pagani and M. Reuter, Ann. Phys. (Amsterdam) 411, 167972 (2019).

[38] O. J. Rosten, Phys. Rep. 511, 177 (2012).

[39] M. Reuter and F. Saueressig, arXiv:0708.1317.

[40] S. Weinberg, in General Relativity: An Einstein Centenary Survey, edited by S. W. Hawking and W. Israel, Chap. 16 (University Press, Cambridge, 1979).

[41] M. Niedermaier, Classical Quant. Grav. 24, R171 (2007).

[42] M. Niedermaier and M. Reuter, Living Rev. Relativity 9, 5 (2006). 
[43] R. Percacci, arXiv:1110.6389.

[44] M. Reuter, Phys. Rev. D 57, 971 (1998).

[45] D. Dou and R. Percacci, Classical Quant. Grav. 15, 3449 (1998).

[46] M. Reuter and H. Weyer, J. Cosmol. Astropart. Phys. 12 (2004) 001.

[47] N. Christiansen, D. F. Litim, J. M. Pawlowski, and A. Rodigast, Phys. Lett. B 728, 114 (2014).

[48] J. Biemans, A. Platania, and F. Saueressig, Phys. Rev. D 95, 086013 (2017).

[49] C. Wetterich, Phys. Lett. B 773, 6 (2017).

[50] F. Canales, B. Koch, C. Laporte, and A. Rincon, J. Cosmol. Astropart. Phys. 01 (2020) 021.

[51] W. Souma, Prog. Theor. Phys. 102, 181 (1999).

[52] O. Lauscher and M. Reuter, Phys. Rev. D 65, 025013 (2001).

[53] M. Reuter and F. Saueressig, Phys. Rev. D 65, 065016 (2002).

[54] D. F. Litim, Phys. Rev. Lett. 92, 201301 (2004).

[55] P. Fischer and D. F. Litim, Phys. Lett. B 638, 497 (2006).

[56] A. Codello and R. Percacci, Phys. Rev. Lett. 97, 221301 (2006).

[57] R. Percacci and D. Perini, Phys. Rev. D 67, 081503 (2003).

[58] R. Percacci and D. Perini, Phys. Rev. D 68, 044018 (2003).

[59] A. Eichhorn and H. Gies, New J. Phys. 13, 125012 (2011).

[60] A. Eichhorn, Phys. Rev. D 86, 105021 (2012).

[61] P. Doná, A. Eichhorn, and R. Percacci, Phys. Rev. D 89, 084035 (2014).

[62] P. Doná, A. Eichhorn, and R. Percacci, Can. J. Phys. 93, 988 (2015).

[63] P. Doná, A. Eichhorn, P. Labus, and R. Percacci, Phys. Rev. D 93, 044049 (2016); 93, 129904(E) (2016).

[64] A. Eichhorn, Found. Phys. 48, 1407 (2018).

[65] S. R. Coleman and E. J. Weinberg, Phys. Rev. D 7, 1888 (1973).

[66] K. A. Meissner and H. Nicolai, Phys. Lett. B 648, 312 (2007).

[67] J. Fatelo, J. Gerard, T. Hambye, and J. Weyers, Phys. Rev. Lett. 74, 492 (1995).

[68] M. Chaichian, R. Gonzalez Felipe, and K. Huitu, Phys. Lett. B 363, 101 (1995).

[69] K. G. Wilson, Rev. Mod. Phys. 47, 773 (1975).

[70] S. Domazet and H. Stefancic, Classical Quant. Grav. 29, 235005 (2012).

[71] T. R. Morris, J. High Energy Phys. 11 (2016) 160.

[72] B. Koch, I. A. Reyes, and Á. Rincón, Classical Quant. Grav. 33, 225010 (2016).

[73] Á. Rincón, B. Koch, and I. Reyes, J. Phys. Conf. Ser. 831, 012007 (2017).

[74] Á. Rincón, E. Contreras, P. Bargueño, B. Koch, G. Panotopoulos, and A. Hernández-Arboleda, Eur. Phys. J. C 77, 494 (2017).

[75] Á. Rincón and B. Koch, Eur. Phys. J. C 78, 1022 (2018).

[76] A. Platania, Universe 5, 189 (2019).

[77] M.E. Peskin and D. V. Schroeder, An Introduction to Quantum Field Theory (Addison-Wesley, Reading, 1995).

[78] S. P. Robinson and F. Wilczek, Phys. Rev. Lett. 96, 231601 (2006).
[79] D. Ebert, J. Plefka, and A. Rodigast, Phys. Lett. B 660, 579 (2008).

[80] Y. Tang and Y. L. Wu, Commun. Theor. Phys. 54, 1040 (2010).

[81] D. J. Toms, Phys. Rev. Lett. 101, 131301 (2008).

[82] D. Ebert, J. Plefka, and A. Rodigast, J. High Energy Phys. 02 (2009) 028.

[83] A. Rodigast and T. Schuster, Phys. Rev. Lett. 104, 081301 (2010).

[84] O. Zanusso, L. Zambelli, G. P. Vacca, and R. Percacci, Phys. Lett. B 689, 90 (2010).

[85] M. M. Anber, J. F. Donoghue, and M. El-Houssieny, Phys. Rev. D 83, 124003 (2011).

[86] D. J. Toms, Phys. Rev. D 84, 084016 (2011).

[87] G. Narain and R. Anishetty, J. High Energy Phys. 10 (2013) 203.

[88] M. T. Grisaru, M. Rocek, and W. Siegel, Phys. Rev. Lett. 45, 1063 (1980).

[89] S. Mandelstam, Nucl. Phys. B213, 149 (1983).

[90] D. J. Toms, Nature (London) 468, 56 (2010).

[91] J. C. C. Felipe, L. C. T. Brito, M. Sampaio, and M. C. Nemes, Phys. Lett. B 700, 86 (2011).

[92] J. C. C. Felipe, L. A. Cabral, L. C. T. Brito, M. Sampaio, and M. C. Nemes, Mod. Phys. Lett. A 28, 1350078 (2013).

[93] A. R. Pietrykowski, Phys. Rev. Lett. 98, 061801 (2007).

[94] D. J. Toms, Phys. Rev. D 76, 045015 (2007).

[95] B. Charneski, A. C. Lehum, and A. J. da Silva, Phys. Rev. D 90, 127702 (2014).

[96] P. J. Mohr, D. B. Newell, and B. N. Taylor, Rev. Mod. Phys. 88, 035009 (2016).

[97] D. M. Webber et al. (MuLan Collaboration), Phys. Rev. Lett. 106, 041803 (2011); 106, 079901 (2011).

[98] T. R. Morris, Int. J. Mod. Phys. A 09, 2411 (1994).

[99] B. Delamotte, Lect. Notes Phys. 852, 49 (2012).

[100] H. Gies, Lect. Notes Phys. 852, 287 (2012).

[101] G. Aad et al. (ATLAS Collaboration), Phys. Lett. B 716, 1 (2012).

[102] S. Chatrchyan et al. (CMS Collaboration), Phys. Lett. B 716, 30 (2012).

[103] F. Vissani, Phys. Rev. D 57, 7027 (1998).

[104] R. Foot, A. Kobakhidze, K. L. McDonald, and R. R. Volkas, Phys. Rev. D 76, 075014 (2007).

[105] R. Foot, A. Kobakhidze, K. L. McDonald, and R. R. Volkas, Phys. Rev. D 77, 035006 (2008).

[106] A. Grinbaum, Found. Phys. 42, 615 (2012).

[107] C. Wetterich, Phys. Lett. B 718, 573 (2012).

[108] A. R. Vieira, B. Hiller, M. C. Nemes, and M. D. R. Sampaio, Int. J. Theor. Phys. 52, 3494 (2013).

[109] M. Farina, D. Pappadopulo, and A. Strumia, J. High Energy Phys. 08 (2013) 022.

[110] M. Heikinheimo, A. Racioppi, M. Raidal, C. Spethmann, and K. Tuominen, Mod. Phys. Lett. A 29, 1450077 (2014).

[111] G. Marques Tavares, M. Schmaltz, and W. Skiba, Phys. Rev. D 89, 015009 (2014).

[112] A. Farzinnia, H. J. He, and J. Ren, Phys. Lett. B 727, 141 (2013).

[113] A. Kobakhidze and K. L. McDonald, J. High Energy Phys. 07 (2014) 155.

[114] N. Haba, H. Ishida, R. Takahashi, and Y. Yamaguchi, Nucl. Phys. B900, 244 (2015). 
[115] N. Craig, A. Katz, M. Strassler, and R. Sundrum, J. High Energy Phys. 07 (2015) 105.

[116] F. Jegerlehner, arXiv:1503.00809.

[117] G. M. Pelaggi, F. Sannino, A. Strumia, and E. Vigiani, Front. Phys. 5, 49 (2017).

[118] G. F. Giudice, Proc. Sci., EPS-HEP2013 (2013) 163 [arXiv:1307.7879].

[119] S. Hossenfelder, Synthese, https://doi.org/10.1007/ s11229-019-02377-5 (2019).

[120] M. J. G. Veltman, Acta Phys. Pol. B 12, 437 (1981).

[121] P. Minkowski, Phys. Lett. 67B, 421 (1977).

[122] R. N. Mohapatra and G. Senjanovic, Phys. Rev. Lett. 44, 912 (1980).

[123] J. Schechter and J. W. F. Valle, Phys. Rev. D 22, 2227 (1980).

[124] M. Cirelli, N. Fornengo, and A. Strumia, Nucl. Phys. B753, 178 (2006).
[125] C. P. Burgess, M. Pospelov, and T. ter Veldhuis, Nucl. Phys. B619, 709 (2001).

[126] M. Shaposhnikov and C. Wetterich, Phys. Lett. B 683, 196 (2010).

[127] M. Shaposhnikov, arXiv:0708.3550.

[128] S. L. Glashow, Nucl. Phys. 22, 579 (1961).

[129] A. Salam, Conf. Proc. C 680519, 367 (1968).

[130] S. Weinberg, Phys. Rev. Lett. 19, 1264 (1967).

[131] A. Eichhorn and F. Versteegen, J. High Energy Phys. 01 (2018) 030.

[132] N. Alkofer and F. Saueressig, Ann. Phys. (Amsterdam) 396, 173 (2018).

[133] H. Gies, B. Knorr, and S. Lippoldt, Phys. Rev. D 92 , 084020 (2015).

[134] A. Codello, R. Percacci, and C. Rahmede, Ann. Phys. (Amsterdam) 324, 414 (2009).

[135] P. Dona and R. Percacci, Phys. Rev. D 87, 045002 (2013). 